\title{
Juergen Schoeftner \\ Bending moment tracking and the reduction of the axial stress in vibrating beams by piezoelectric actuation
}

Received: 20 February 2017 / Published online: 25 July 2017

(C) The Author(s) 2017. This article is an open access publication

\begin{abstract}
This contribution focuses on bending moment tracking in slender beam-type structures that are equipped with piezoelectric actuators. Bending moments are associated with the axial stress, which is the dominant stress component of laterally excited beam structures. If the maximum value exceeds a certain tensile stress limit, the structures will crack or be irreparably damaged. In the present contribution, a piezoelectric bimorph beam is considered and it is investigated in which manner the piezoelectric actuation devices have to be distributed along the beam length, such that a certain bending moment distribution is obtained. This is called bending moment tracking. First, the basic equations of a piezoelectric bimorph beam are recalled and the differential equations for the bending moment are derived. Then a positive semi-definite integral depending on the error of the bending moment is defined, which is the difference between the actual and the desired bending moment. The results of the derivations are conditions for the eigencurvature due to the piezoelectric actuation, such that a certain bending moment distribution is achieved. Approximate solutions for the eigencurvature are also presented for the lower- and for the high-frequency domain. The theory is verified by a support-excited piezoelectric bimorph. First, the frequency response curves for the deflection, the bending moment and the axial stresses are calculated. Then the responses due to a sinusoidal excitation are computed, showing that the suggested control algorithm enables the reduction of the bending moment and also of the axial stress in a satisfactory manner.
\end{abstract}

\section{Introduction}

Engineering mechanics deals with the solution of engineering problems in terms of displacements, strains and stresses. The stress and the displacement fields may be determined from the partial differential equations if external loadings or eigenstrain sources are prescribed. The content of this contribution is restricted to the linear theory of elasticity. For the basics, the reader is referred to Hetnarski and Ignaczak [1], Gurtin [2] Lurie [3].

One possibility of eigenstrain sources is due to the piezoelectric effect. This effect is well established in control engineering, sensing and structural health monitoring, see e.g. Moheimani and Fleming [4]. A further overview of piezoelectricity is given by Crawley [5], Miu [6] and Tzou [7]. The notion of eigenstrain was first introduced by Mura [8] and means a non-elastic strain, e.g. an initial, plastic or misfit strain. Non-elastic strains can also be caused by thermal variations or by piezoelectricity. In the last three decades, piezoelectric transducers became popular candidates for vibration control or displacement tracking, see e.g. Schoeftner and Krommer [9], Krommer and Irschik [10,11], Han et al. [12] or Benjeddou and Deü [13].

One particular application of displacement tracking is known under the notion shape control, when the displacement to be tracked is nullified. A literature review on this topic is presented by Irschik [14]. Further

J. Schoeftner $(\varangle)$

Institute of Technical Mechanics, Johannes Kepler University of Linz, Altenbergerstr. 69, 4020 Linz, Austria

E-mail: juergen.schoeftner@jku.at

Tel.: +43-70-2468-6298

Fax: +43-70-2468-6282 
discussions on shape control can be found in Hubbard and Burke [15], Hafka and Adelman [16], Irschik et al. [17, 18], Nader [19], Irschik and Pichler et al. [20], Schoeftner et al. [21-23], Austin et al. [24] and Agrawal and Treanor [25]. Displacement tracking is a feed-forward control method, where one asks for the eigenstrain sources in order to obtain a certain deflection. This means that one faces an inversely posed problem, in contrary to the ordinary problem, where one wants to calculate the displacement when imposed forces and moments are known. For some recent formulations, see Irschik et al. [26].

It is obvious that one may try to track the stress field instead of the displacement field: replacing the displacement in the equations of motion by the stress, one may ask how to obtain a certain stress distribution. Then the notion of stress tracking is used. Surprisingly, stress annihilation or stress reduction has been rarely treated in such a systematic way as displacement tracking or shape control, although mechanical stress is understood as the driving factor for the collapse, material fatigue and damage of structures when exceeding a certain stress level. Irschik [27] discusses how to achieve a certain stress or displacement fields when prescribing the spatial and temporal control actuation. The link between shape control and stress tracking is presented in Irschik et al. [28], where it was found that the stress distribution in a body, where the displacements are completely annihilated by eigenstrain actuation, is equal to the quasi-static stress distribution caused by the external load.

Here the focus lies on the control of the bending moment which represents the resultant moment of the axial stress distribution. The paper is organized as follows:

After recalling the fundamental relations for a slender beam (the equations of motion and the constitutive relations), a differential equation is derived for the bending moment in Sect. 2. In Sect. 3, the error displacement and the error bending moment are defined, which are the differences between the actual and the desired displacement and bending moment, respectively. A semi-positive definite time-dependent integral is defined, which involves the squares of the derivatives of the error moment with respect to the time and the spatial coordinate. Stating that the time derivative of this integral is zero, conditions for the control eigenstrain are obtained. In Sect. 4, the derived control method is illustrated: a support-excited beam is studied and the desired bending moment distribution is realized according to the derived conditions for the eigencurvature. Finally, an outlook is given on approximate solutions in the lower or in the higher frequency range, which may become important for the practical realization. Additionally, it is pointed out that a reduction of the bending moment with this technique means also a reduction of the axial stress.

\section{Equations of a piezoelectric beam}

The partial differential equation for the lateral vibration $w(x, t)$ of a beam can be written as, see Ziegler [29],

$$
m \ddot{w}(x, t)=M_{, x x}(x, t)+b(x, t) .
$$

The mass density per unit length, the second derivative of the bending moment and the distributed load are denoted by $m, M_{, x x}(x, t)$ and $b(x, t)$, respectively. The axial coordinate is $x$ and the time is $t$. The derivative with respect to the axial coordinate and the time derivative of an arbitrary function $g(x, t)$ are denoted by $g_{, x}(x, t)$ and $\dot{g}(x, t)$. For thermomechanical, piezoelectric or elastomagnetic material, the bending moment reads, see Schoeftner et al. [23,30],

$$
M(x, t)=-K_{\mathrm{M}}\left[w_{, x x}(x, t)+\kappa_{\mathrm{c}}(x, t)\right],
$$

where the bending stiffness is $K_{\mathrm{M}}$. The second term within the square bracket is influenced by the eigencurvature $\kappa_{\mathrm{c}}(x, t)$. In case of a piezoelectric bimorph, one finds a relation between the eigencurvature and the applied electric voltage $V(x, t)$ (see Schoeftner et al. [23])

$$
\kappa_{\mathrm{c}}(x, t)=-\frac{e_{31} h b}{K_{\mathrm{M}}} V(x, t)
$$

where $e_{31}, h$ and $b$ are the piezoelectric modulus, the height and the width of the piezoelectric layers. Substituting Eq. (2) into Eq. (1), one finds the equation of motion as

$$
m \ddot{w}(x, t)=-K_{\mathrm{M}}\left[w_{, x x x x}(x, t)+\kappa_{\mathrm{c}, x x}(x, t)\right]+b(x, t) .
$$


Differentiation of Eq. (2) with respect to the time twice and rearranging the outcome, one finds

$$
\ddot{w}_{, x x}(x, t)=-\frac{\ddot{M}(x, t)}{K_{\mathrm{M}}}-\ddot{\kappa}_{\mathrm{c}}(x, t) .
$$

Substituting Eq. (5) into Eq. (1), the differential equation for the bending moment is obtained

$$
\frac{m}{K_{\mathrm{M}}} \ddot{M}(x, t)+M_{, x x x x}(x, t)=-m \ddot{\kappa}_{\mathrm{c}}(x, t)-b_{, x x}(x, t) .
$$

\section{Bending moment control and conditions}

The goal of the present contribution is to manipulate the bending moment $M(x, t)$, and, as a consequence, also to reduce the axial stress $\sigma(x, t)$.

In a first step, the error displacement is introduced,

$$
w_{e}(x, t):=w_{f}(x, t)+w_{c}(x, t)-w_{d}(x, t),
$$

and the error bending moment

$$
M_{e}(x, t):=M_{f}(x, t)+M_{c}(x, t)-M_{d}(x, t) .
$$

It is noted that the actual displacement is $w(x, t)=w_{f}(x, t)+w_{c}(x, t)$. In an analogous manner, this also holds for the actual bending moment $M(x, t)=M_{f}(x, t)+M_{c}(x, t)$. The error signals are the differences between the actual signals and the desired values $\left(w_{d}(x, t)\right.$ and $\left.M_{d}(x, t)\right)$. The subscripts $f$ and $c$ mean the additive decomposition of the displacement and the bending moment, caused by either the external forces (subscript $f$ ) or the control eigencurvature (subscript $c$ ).

Based on Eqs. (7) and (8), one defines the error integral as a function of the error bending moment:

$$
I_{\mathrm{M}}(t)=\int_{x}\left(K_{\mathrm{M}} M_{e, x x}^{2}+m \dot{M}_{e}^{2}\right) \mathrm{d} x .
$$

Since the mass density per unit length $m$ and the bending stiffness $K_{\mathrm{M}}$ are semi-positive definite properties, also the error integral is positive definite at every time instant $t$. In a first step, it is shown under which circumstances the integral remains time independent, i.e. $\dot{I}_{\mathrm{M}}(t)=0$ or $I_{\mathrm{M}}(t)=I_{\mathrm{M}}(0)$. In a second step, it is shown that if the integral vanishes from the beginning, i.e. if $I_{\mathrm{M}}(0)=0$, this also means that the error bending moment is zero $M_{e}(x, t)=0$ or that the actual and the desired bending moments are equal $M_{f}(x, t)+M_{c}(x, t)=M_{d}(x, t)$.

\subsection{First step: $\dot{I}_{\mathrm{M}}(t)=0$}

Differentiating Eq. (9) with respect to time yields

$$
\frac{\dot{I}_{\mathrm{M}}(t)}{2}=\int_{x}\left(K_{\mathrm{M}} M_{e, x x} \dot{M}_{e, x x}+m \dot{M}_{e} \ddot{M}_{e}\right) \mathrm{d} x .
$$

Reformulating the first term on the right-hand side using integration by parts,

$$
M_{e, x x} \dot{M}_{e, x x}=\left(M_{e, x x} \dot{M}_{e, x}\right)_{, x}-\left(M_{e, x x x} \dot{M}_{e}\right)_{, x}+\left(M_{e, x x x x} \dot{M}_{e}\right),
$$

and substituting Eqs. (6), (8) and (11) into Eq. (10), it follows that

$$
\begin{aligned}
\frac{\dot{I}_{\mathrm{M}}(t)}{2}= & \int_{x}\left[K_{\mathrm{M}} \dot{M}_{e}\left(-M_{d, x x x x}-\frac{m}{K_{\mathrm{M}}} \ddot{M}_{d}-m \ddot{\kappa}_{\mathrm{c}}-b_{, x x}\right)\right] \mathrm{d} x \\
& +\left.K_{\mathrm{M}}\left[M_{e, x x} \dot{M}_{e, x}-M_{e, x x x} \dot{M}_{e}\right]\right|_{\partial B_{k} \cup \partial B_{d}} .
\end{aligned}
$$


The kinematic and dynamic boundary terms are indicated by $\partial B_{k}$ and $\partial B_{d}$. The last line in Eq. (12) can be rewritten as

$$
\begin{aligned}
& {\left.\left[M_{e, x x} \dot{M}_{e, x}-M_{e, x x x} \dot{M}_{e}\right]\right|_{\partial B_{k} \cup \partial B_{d}}} \\
& =\left.\left[M_{e, x x}\left(\dot{Q}_{f}+\dot{Q}_{c}-\dot{Q}_{d}\right)-M_{e, x x x}\left(\dot{M}_{f}+\dot{M}_{c}-\dot{M}_{d}\right)\right]\right|_{\partial B_{d}} \\
& \quad+\dot{M}_{e, x}\left(m \ddot{w}-b-M_{d, x x}\right)-\left.\dot{M}_{e}\left(m \ddot{w}_{, x}-b_{, x}-M_{d, x x x}\right)\right|_{\partial B_{k}}
\end{aligned}
$$

The time derivative of the error integral $\dot{I}_{\mathrm{M}}(t)$ vanishes, if the integrand vanishes in Eq. (12), i.e. a condition for the eigencurvature $\kappa_{\mathrm{c}}(x, t)$ (=the control input) is obtained from the first term on the right-hand side:

$$
\ddot{\kappa}_{\mathrm{c}}=-\frac{1}{m}\left(b+M_{d, x x}\right)_{, x x}-\frac{1}{K_{\mathrm{M}}} \ddot{M}_{d} .
$$

Time integration of the control input condition (14) yields

$$
\kappa_{\mathrm{c}}=\iint\left[-\frac{1}{m}\left(b+M_{d, x x}\right)_{, x x}\right] \mathrm{d} t \mathrm{~d} t-\frac{1}{K_{\mathrm{M}}} M_{d}+K_{1}(x) t+K_{0}(x) .
$$

The determination of the functions $K_{1}(x)$ and $K_{0}(x)$ is discussed in Sect. 3.2.

According to Eq. (13), the following relations must hold at the geometric (kinematic) $\partial B_{k}$ and/or the natural (dynamic) $\partial B_{d}$ boundaries

$$
\begin{array}{ll}
\partial B_{d}: & \dot{M}_{c}=-\dot{M}_{f}+\dot{M}_{d}, \\
& \dot{M}_{c, x}=\dot{Q}_{c}=-\dot{Q}_{f}+\dot{Q}_{d}, \\
\partial B_{k}: & \ddot{w}_{c}=-\ddot{w}_{f}+\frac{1}{m}\left(b+M_{d, x x}\right), \\
& \ddot{w}_{c, x}=-\ddot{w}_{f, x}+\frac{1}{m}\left(b_{, x}+M_{d, x x x}\right) .
\end{array}
$$

\subsection{Second step: show that $I_{\mathrm{M}}(0)=0$}

Satisfying the conditions (16)-(19) and (14) yields that the error integral (9) remains constant, i.e. if one knows its value at the beginning, then the value is known for all times: $I_{\mathrm{M}}(t)=I_{\mathrm{M}}(0)$.

Substituting Eqs. (8) and (2) into the error integral (9), one finds

$$
\begin{aligned}
I_{\mathrm{M}}(0)= & \int_{x} K_{\mathrm{M}}\left[-K_{\mathrm{M}}\left(w_{f, x x x x}+w_{c, x x x x}+\kappa_{\mathrm{c}, x x}\right)-M_{d, x x}\right]^{2} \mathrm{~d} x \\
& +\int_{x} m\left[-K_{\mathrm{M}}\left(\dot{w}_{f, x x}+\dot{w}_{c, x x}+\dot{\kappa}_{\mathrm{c}}\right)-\dot{M}_{d}\right]^{2} \mathrm{~d} x .
\end{aligned}
$$

The square brackets represent $M_{e, x x}(x, 0)$ and $\dot{M}_{e}(x, 0)$, see Eq. (8). The condition $I_{\mathrm{M}}(0)=0$ is only fulfilled if the error moment is a linear function in $x$, i.e. $M_{e}(x, t)=a+b x$. Since the goal is to nullify the error bending moment $M_{e}(x, t)=0$ (which is a stricter condition than demanding $M_{e, x x}(x, 0)=\dot{M}_{e}(x, 0)=0$ ), the coefficients $a$ and $b$ have to disappear. This is achieved by adjusting the initial control input in such a manner that the following relations are satisfied:

$$
\begin{aligned}
t=0: \quad M_{e}(x, 0)=0 & \rightarrow K_{\mathrm{M}} w_{c, x x}(x, 0)+K_{\mathrm{M} \kappa_{\mathrm{c}}}(x, 0) \\
& =-K_{\mathrm{M}} w_{f, x x}(x, 0)-M_{d}(x, 0), \\
\dot{M}_{e}(x, 0)=0 & \rightarrow K_{\mathrm{M}} \dot{w}_{c, x x}(x, 0)+K_{\mathrm{M}} \dot{\kappa}_{\mathrm{c}}(x, 0) \\
& =-K_{\mathrm{M}} \dot{w}_{f, x x}(x, 0)-\dot{M}_{d}(x, 0) .
\end{aligned}
$$

These two equations are used to compute the $x$-dependent functions $K_{1}(x)$ and $K_{0}(x)$ in Eq. (15) for the eigencurvature $\kappa_{\mathrm{c}}(x, t)$, because the initial conditions of the deflection $w(x, 0), \dot{w}(x, 0)$, the desired bending moment $M_{d}(x, 0)$ and the external loading $b(x, 0)$ are known at the beginning. 
3.3 Analytical solution of the deflection in case of bending moment tracking

Inserting the eigencurvature solution (15) into the equations of motion (4), the analytical solution for the displacement is

$$
w(x, t)=\iint \frac{1}{m}\left[b(x, t)+M_{d, x x}(x, t)\right] \mathrm{d} t \mathrm{~d} t-\iint\left[K_{1}(x) t+K_{0}(x)\right] \mathrm{d} x \mathrm{~d} x .
$$

It can be easily checked that Eq. (23) satisfies

- the equation of motion Eq. (4),

- the kinematic and dynamic boundary conditions Eqs. (16)-(19) and

- the initial conditions Eqs. (21)-(22).

\section{Example: bending moment control of a support-excited cantilever}

In this section, the theory for bending moment control is validated with a simple example: a piezoelectric beam is considered, whose vertical motion is prescribed at the left end $w(x, t)=w_{\mathrm{p}}(t)$ (see Fig. 1a). An external load is not present, $b(x, t)=0$. The parameters for the piezoelectric bimorph are listed in Table 1.

The desired bending moment is assumed to be

$$
M_{d}(x, t)=m \ddot{w}_{\mathrm{p}}(t) g(x),
$$

where the spatial function $g(x)$ (see Fig. 1b) is taken as

$$
g(x)=\left(-\frac{\bar{x}}{3}+\frac{\bar{x}^{2}}{2}-\frac{\bar{x}^{4}}{6}\right) l^{2} \text { and } \bar{x}=\frac{x}{l} .
$$

(a) upper

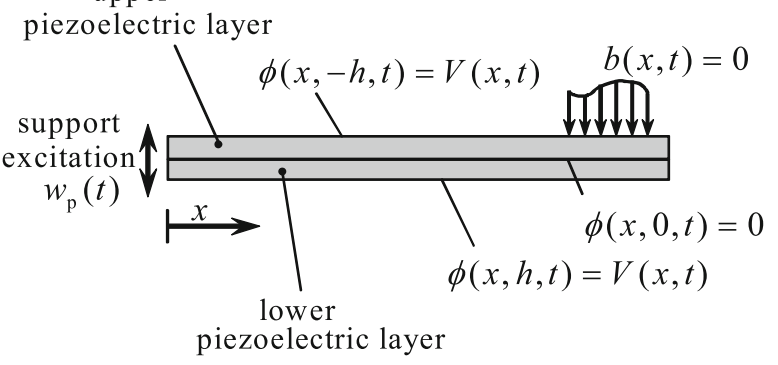

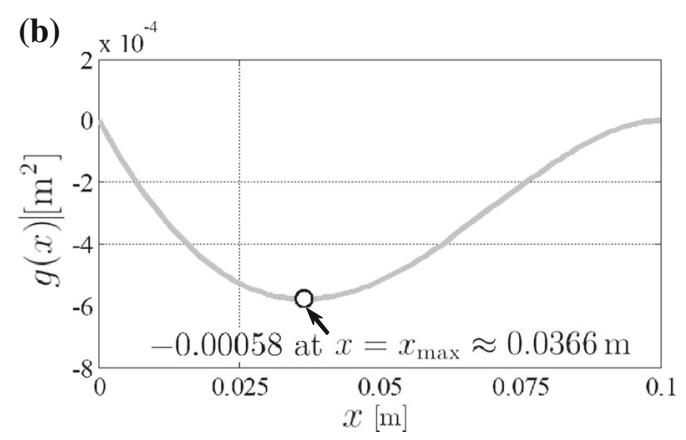

Fig. 1 Support-excited piezoelectric cantilever (a); spatial distribution $g(x)$ of the desired bending moment $M_{d}(x, t)$, see Eq. (24) (b)

Table 1 Parameters for the numerical examples

\begin{tabular}{llll}
\hline Variable & Unit & Value & Description \\
\hline$\rho$ & $\mathrm{kgm}^{-3}$ & 7750 & Density \\
$h$ & $\mathrm{~m}$ & $1.00 \times 10^{-3}$ & Thickness (of each layer) \\
$b$ & $\mathrm{~m}$ & $5.00 \times 10^{-2}$ & Width \\
$e_{31}$ & $\mathrm{Asm}^{-2}$ & -10.43 & Piezoelectric modulus \\
$C$ & $\mathrm{Nm}^{-2}$ & $6.10 \times 10^{10}$ & Young's modulus \\
$\kappa_{33}$ & $\mathrm{AsV}^{-1} \mathrm{~m}^{-1}$ & $1.33 \times 10^{-8}$ & Strain-free permittivity \\
$l$ & $\mathrm{~m}$ & 0.1 & Length \\
$d$ & - & 0.001 & Modal damping (of each mode) \\
$K_{\mathrm{M}}=\left(\frac{2}{3} C+\frac{e_{31}^{2}}{6 \kappa_{33}}\right) h^{3} b$ & $\mathrm{Nm}^{2}$ & 2.10 & Length \\
$m=2 \rho h b$ & $\mathrm{kgm}^{-1}$ & 0.775 & Mass density per unit length \\
$w_{0}$ & $\mathrm{~m}$ & 0.0001 & Amplitude of prescribed boundary excitation \\
\hline
\end{tabular}


This means that the maximum value of bending moment will occur at $x_{\max } \approx 0.0366 \mathrm{~m}$, where the function has its maximum $\left|g\left(x_{\max }\right)\right|=5.8 \times 10^{-4} \mathrm{~m}^{2}$. At the free end at $x=l$, the dynamic boundary condition with the control method is automatically fulfilled, see Eqs. (16), (17), (24) and (25):

$$
\begin{aligned}
& \dot{M}_{d}(l, t)=0 \\
& \dot{Q}_{d}(l, t)=0 .
\end{aligned}
$$

For the vertically excited end, the desired bending moment is related to the transient excitation and thus cannot be chosen arbitrarily due to the kinematic boundary conditions in Eqs. (18) and (19). At $x=0$, the following relations hold:

$$
\begin{aligned}
\ddot{w}(0, t) & =\frac{1}{m} M_{\mathrm{d}, x x}(0, t) \underbrace{=\ddot{w}_{\mathrm{p}}(t)}_{\text {with Eqs. (24) and (25) }}, \\
\ddot{w}_{, x}(0, t) & =\frac{1}{m} M_{\mathrm{d}, x x x}(0, t) \underbrace{=0}_{\text {with Eqs. (24) and (25) }} .
\end{aligned}
$$

First, a harmonic analysis (Sect. 4.2) is performed and then the transient excitation is assumed to be sinusoidal with $w_{\mathrm{p}}(t)=w_{0}[1-\cos (\omega t)]$ (Sect. 4.3). lated:

The following four notations are used for the uncontrolled and controlled configurations, which are simu-

1. No control The control eigencurvature is zero:

$$
\kappa_{\mathrm{c}}(x, t)=0 .
$$

2. Bending moment control The eigencurvature is chosen according to Eq. (15):

$$
\kappa_{\mathrm{c}}(x, t)=\frac{4}{l^{2}} w_{\mathrm{p}}(t)-\frac{m}{K_{\mathrm{M}}} l^{2}\left(-\frac{\bar{x}}{3}+\frac{\bar{x}^{2}}{2}-\frac{\bar{x}^{4}}{6}\right) \ddot{w}_{\mathrm{p}}(t) .
$$

Note that the functions $K_{0}(x)$ and $K_{1}(x)$ will vanish if one assumes the beam to rest at the beginning $w(x, 0)=\dot{w}(x, 0)=0$, see Eqs. (15), (21) and (22)

3. Bending moment control with the static term only The second time derivative in Eq. (31) is neglected:

$$
\kappa_{\mathrm{c}}(x, t)=\frac{4}{l^{2}} w_{\mathrm{p}}(t) \quad(\text { control static }) .
$$

4. Bending moment control with the dynamic term only The first term in Eq. (31) is neglected:

$$
\kappa_{\mathrm{c}}(x, t)=-\frac{m}{K_{\mathrm{M}}} l^{2}\left(-\frac{\bar{x}}{3}+\frac{\bar{x}^{2}}{2}-\frac{\bar{x}^{4}}{6}\right) \ddot{w}_{\mathrm{p}}(t) \quad \text { (control dynamic) } .
$$

Since damping is considered (modal damping of $0.1 \%$ for each mode is assumed), the beam problem is solved by means of numerical methods, where the beam is discretized into 50 finite elements. The interpolation functions for each element are Hermite ansatz functions, see also Schoeftner and Buchberger [31]. The analytical solution of the displacement in case of bending moment control is given in Eq. (23) for the undamped configuration. For the example, the analytical displacement reads

$$
w(x, t)=\left(1-2 \bar{x}^{2}\right) w_{\mathrm{p}}(t),
$$

see Eq. (23). Defining the relative displacement as the difference of the absolute displacement and the boundary excitation, one finds

$$
w_{\text {rel }}(x, t)=-2 \bar{x}^{2} w_{\mathrm{p}}(t) .
$$

It is noted that damping is neglected in analytical solution (34), but since the modal damping coefficient is low, it is expected that the numerical and analytical solutions hardly will differ. In order to check the results, one may insert the analytical solution for displacement (34) and for eigencurvature (31) into the constitutive relation on beam level (2) and obtains the desired bending moment distribution, cf. (24) and (25). 


\subsection{Axial stress distribution in case of bending moment control}

As was pointed out in the introduction, the local stress is responsible for the collapse of structures. In this section, it is shown that the maximum stress is also reduced if the bending moment control technique is applied. The axial stress $\sigma_{x x}(x, z, t)$ requires the knowledge of the second derivative of the displacement $w_{, x x}$ and the electric field $E_{z}(x, t)$ :

$$
\sigma_{x x}(x, z, t)=-C z w_{, x x}(x, t)-e_{31} E_{z}(x, t) .
$$

By means of the relations for piezoelectric beams given, e.g. in Schoeftner et al. $[23,30]$, the stress can be calculated as follows: the electric field is related to the $z$-derivative of the electric potential $\phi(x, z, t)$,

$$
E_{z}(x, t)=-\phi_{, z}(x, z, t),
$$

and the potential depends on the electric voltage across the electrodes:

$$
\phi(x, z, t)= \pm \frac{V(x, t)}{h} z+\frac{e_{31}}{\kappa_{33}}\left( \pm \frac{h}{2} z-\frac{z^{2}}{2}\right) w_{, x x}(x, t) .
$$

The plus sign corresponds to the lower layer $(0 \leq z \leq h)$, whereas the minus sign corresponds to the upper layer $(-h \leq z<0)$. Combining the eigencurvature solution (31) with (3), the actuation voltage is obtained as

$$
V(x, t)=-\frac{K_{\mathrm{M}}}{e_{31} h b}\left[\frac{4}{l^{2}} w_{\mathrm{p}}(t)-\frac{m}{K_{\mathrm{M}}} l^{2}\left(-\frac{\bar{x}}{3}+\frac{\bar{x}^{2}}{2}-\frac{\bar{x}^{4}}{6}\right) \ddot{w}_{\mathrm{p}}(t)\right] .
$$

Inserting Eqs. (34), (37)-(39) into (36), one finds for the axial stress

$$
\begin{aligned}
\sigma_{x x}(x, z, t)= & \frac{4}{l^{2}} w_{\mathrm{p}}(t)\left[C z-\frac{e_{31}^{2}}{\kappa_{33}}\left( \pm \frac{h}{2}-z\right) \mp \frac{K_{\mathrm{M}}}{h^{2} b}\right] \\
& \pm \frac{m l^{2}}{h^{2} b}\left(-\frac{\bar{x}}{3}+\frac{\bar{x}^{2}}{2}-\frac{\bar{x}^{4}}{6}\right) \ddot{w}_{\mathrm{p}}(t) .
\end{aligned}
$$

Substituting for the bending stiffness $K_{\mathrm{M}}=\left(\frac{2}{3} C+\frac{e_{31}^{2}}{6 \kappa_{33}}\right) h^{3} b$ (see Table 1) and transforming the result into the frequency domain (indicated by the hat-symbol $\hat{\sigma}_{x x}$ ), one finds the axial stress for the lower and the upper layer

$$
\hat{\sigma}_{x x}(x, z, \omega)=[\frac{4}{l^{2}}\left(C+\frac{e_{31}^{2}}{\kappa_{33}}\right)\left(z \mp \frac{2}{3} h\right) \mp \frac{m l^{2}}{h^{2} b} \underbrace{\left(-\frac{\bar{x}}{3}+\frac{\bar{x}^{2}}{2}-\frac{\bar{x}^{4}}{6}\right)}_{=g(x) / l^{2}, \text { see Eq. }(25)} \omega^{2}] \hat{w}_{\mathrm{p}} .
$$

Taking into consideration that the function $g(x)$ is negative (see Fig. $1 \mathrm{~b}$ and assuming $\hat{w}_{\mathrm{p}}>0$ ), the maximal tensile stress $\hat{\sigma}_{x x}>0$ for the lower layer occurs at $z=h$ :

$$
\begin{aligned}
\hat{\sigma}_{x x, \max }(x, \omega) & =\hat{\sigma}_{x x}(x, h, \omega) \\
& =\left[\frac{4 h}{3 l^{2}}\left(C+\frac{e_{31}^{2}}{\kappa_{33}}\right)-\frac{m l^{2}}{h^{2} b}\left(-\frac{\bar{x}}{3}+\frac{\bar{x}^{2}}{2}-\frac{\bar{x}^{4}}{6}\right) \omega^{2}\right] \hat{w}_{\mathrm{p}},
\end{aligned}
$$

and for the upper layer at $z=0$ :

$$
\begin{aligned}
\hat{\sigma}_{x x, \max }(x, \omega) & =\hat{\sigma}_{x x}(x, 0, \omega) \\
& =\left[\frac{8 h}{3 l^{2}}\left(C+\frac{e_{31}^{2}}{\kappa_{33}}\right)+\frac{m l^{2}}{h^{2} b}\left(-\frac{\bar{x}}{3}+\frac{\bar{x}^{2}}{2}-\frac{\bar{x}^{4}}{6}\right) \omega^{2}\right] \hat{w}_{\mathrm{p}} .
\end{aligned}
$$


One observes that in the lower frequency domain (i.e. $\omega$ or $f$ is small), the maximum tensile stress occurs in the upper layer at $x=0$ and it reads

$$
\omega \text { is small : } \quad \hat{\sigma}_{x x, \max }(x=0, \omega)=\frac{8 h}{3 l^{2}}\left(C+\frac{e_{31}^{2}}{\kappa_{33}}\right) \hat{w}_{\mathrm{p}} .
$$

In the higher frequency domain (i.e. $\omega$ or $f$ is large), the maximum tensile stress occurs in the lower layer at $x=x_{\max }$ and is proportional to the square of the frequency

$$
\omega \text { is large : } \hat{\sigma}_{x x, \max }\left(x=x_{\max }, \omega\right) \approx \frac{m}{h^{2} b}\left|g\left(x_{\max }\right)\right| \omega^{2} \hat{w}_{\mathrm{p}} .
$$

Comparing the order of magnitudes of the frequency-dependent and frequency-independent terms in Eqs. (42) and (43), one may derive the following characteristic frequency, called the limit frequency:

$$
f^{+} \approx \frac{1}{2 \pi} \sqrt{\frac{2 h^{2}\left(C+\frac{e_{31}^{2}}{\kappa_{33}}\right)}{3 l^{2}\left|g\left(x_{\max }\right)\right| \rho}} \approx 161 \mathrm{~Hz} .
$$

If the excitation frequency is below this limit, the maximum tensile stress is close to Eq. (44). If it is higher, then it is close to Eq. (45), see Fig. 2c.

\subsection{Frequency response curves}

Figures 2 and 3 show the frequency responses of

- The relative displacement $\hat{w}_{\text {rel }}(l)$ (Figs. 2a and 3a),
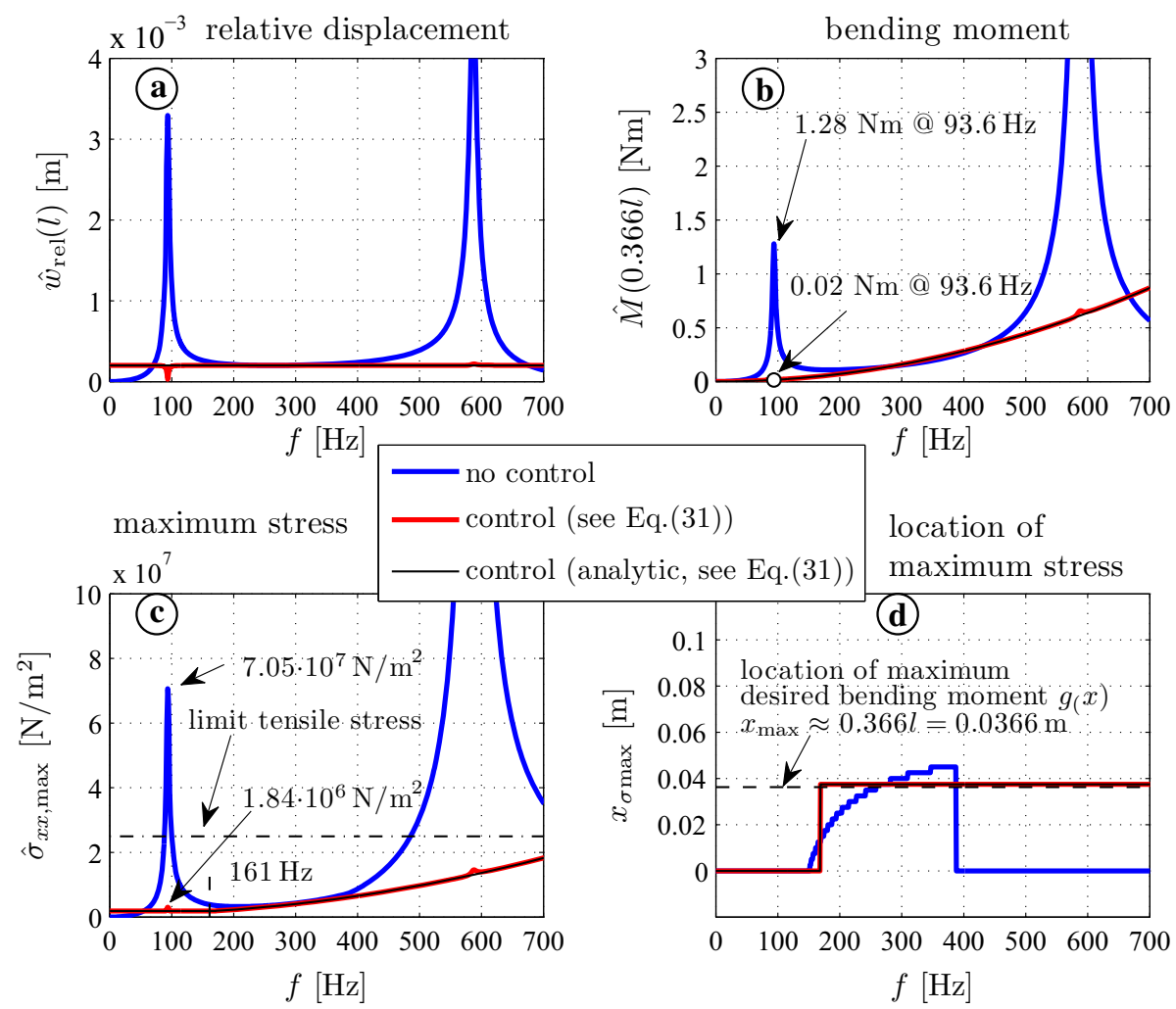

Fig. 2 Frequency response functions of the uncontrolled Eq. (30) and controlled configurations Eq. (31) (relative displacement $\hat{w}_{\text {rel }}(l)(\mathbf{a})$, bending moment $\hat{M}$ at $x=0.366 l(\mathbf{b})$, maximum tensile stress $\hat{\sigma}_{x x \text {, max }}(\mathbf{c})$ and its location (d)) 

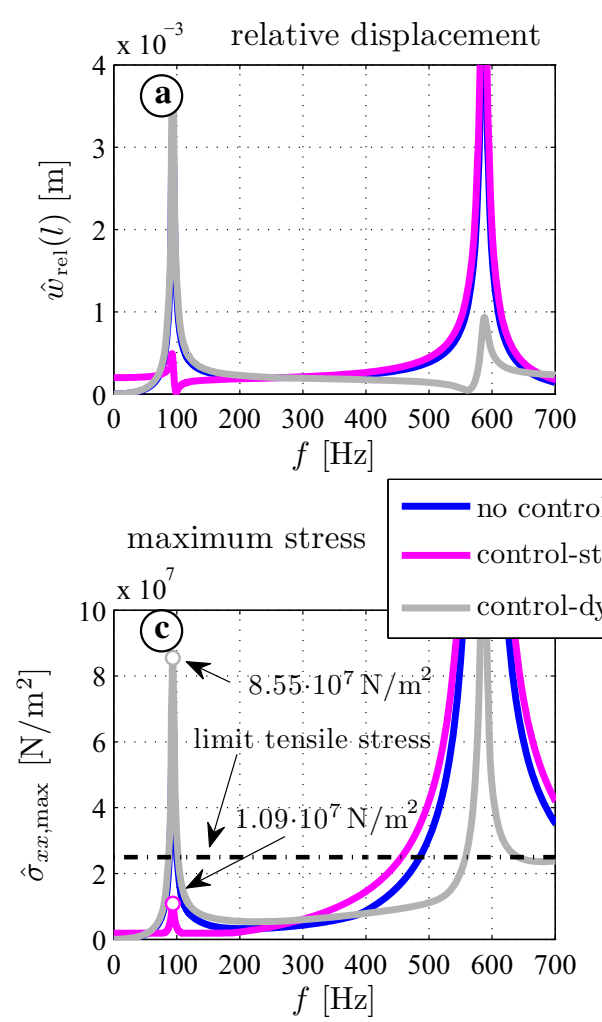

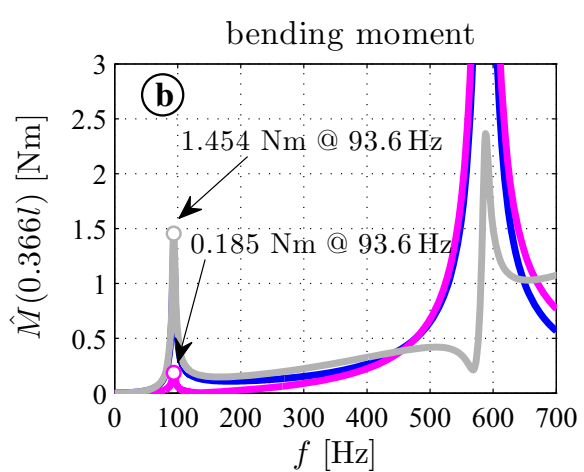

location of maximum stress

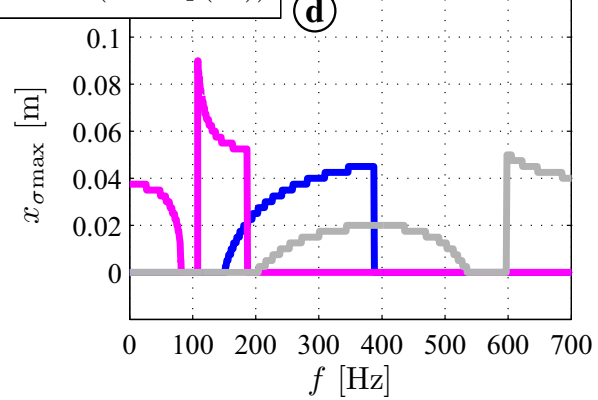

Fig. 3 Frequency response functions of the controlled configurations (control-static Eq. (32) and control-dynamic Eq. (33)) (relative displacement $\hat{w}_{\text {rel }}(l)(\mathbf{a})$, bending moment $\hat{M}$ at $x=0.366 l(\mathbf{b})$, maximum tensile stress $\hat{\sigma}_{x x, \max }(\mathbf{c})$ and its location (d))

- The bending moment $\hat{M}(0.366 l)$ (Figs. $2 \mathrm{~b}$ and $3 \mathrm{~b})$,

- The maximum axial stress $\hat{\sigma}_{x x \text {, max }}$ (Figs. $2 \mathrm{c}$ and $3 \mathrm{c}$ ) and

- The location of the maximum stress $x_{\sigma \max }$ (Figs. $2 \mathrm{~d}$ and $3 \mathrm{~d}$ ),

when the amplitude of the boundary excitation is $\hat{w}_{\mathrm{p}}=0.0001 \mathrm{~m}$. First, the results obtained by the bending moment control (Eq. (31)) are compared to the uncontrolled results (Eq. (30)), see Fig. 2. Then the two alternative control methods (bending moment control with the static (Eq. (32)) or dynamic term only (Eq. (33)) are compared in Fig. 3.

In Fig. 2a, one recognizes the fundamental eigenfrequency $f_{1}=93.6 \mathrm{~Hz}$ and the second eigenfrequency $f_{2}=586.7 \mathrm{~Hz}$ for the uncontrolled configuration (blue). The result for the bending moment (finite element discretization of the controlled case-red) is in very good agreement to the analytical solution (black when no damping is considered): the relative displacement is $\hat{w}_{\text {rel }}(l)=0.0002 \mathrm{~m}$, see Eq. (35).

Figure $2 \mathrm{~b}$ shows the bending moment at $x=0.366 \mathrm{l}$. Close to the eigenfrequencies, the bending moment is drastically reduced. The maximum bending moment for the uncontrolled case at $f \approx 93.6 \mathrm{~Hz}$ is $1.28 \mathrm{Nm}$ and reduced to $0.02 \mathrm{Nm}$. One also observes that in the frequency domain the desired bending moment is a quadratic function $\left|\hat{M}_{d}(x, \omega)\right|=\omega^{2} m \hat{w}_{\mathrm{p}} g(x)$, see also Eq. (24).

The maximum stress results are shown in Fig. 2c. For $f<161 \mathrm{~Hz}$ (note this value corresponds to the limit frequency value stated in Eq. (46)), the maximum value does not depend on the frequency and is constant $\hat{\sigma}_{x x \text {, max }}=1.84 \times 10^{6} \mathrm{Nm}^{-2}$ at $x=0 \mathrm{~m}$, see Eq. (44). This value is much lower than the limit tensile stress for $\sigma_{\text {lim }}=2.5 \times 10^{7} \mathrm{Nm}^{-2}$ (dash-dot black line), which is a typical mechanical strength value of PZT ceramic material, see also [32]. For the uncontrolled case, the stress is $\hat{\sigma}_{x x, \max } \approx 7.05 \times 10^{7} \mathrm{Nm}^{-2}$ at the first eigenfrequency.

For higher frequencies, the stress also increases quadratically (see Eq. (45)) and the maximum stress changes from location $x=0 \mathrm{~m}$ to $x \approx 0.0366 \mathrm{~m}$ at $f=161 \mathrm{~Hz}$, as shown in Fig. $2 \mathrm{~d}$. Within the shown frequency range, the maximum stress does not exceed the stress limit, but due to the quadratic dependency on the frequency, the stress cannot be kept below the maximum stress limit for high frequencies (i.e. $f>750 \mathrm{~Hz}$, results not shown in Fig. 2c). 

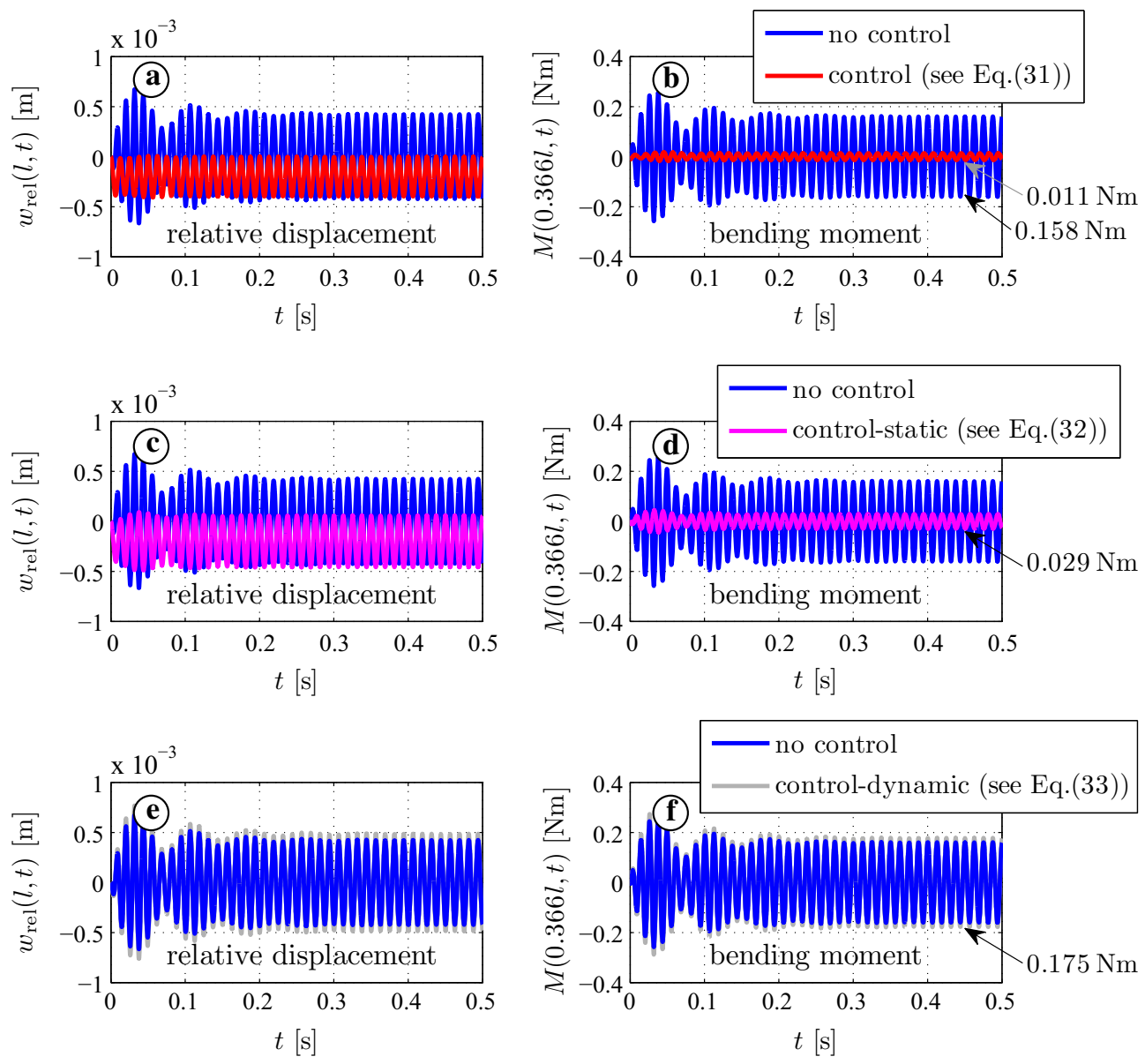

Fig. 4 Transient response due to the support excitation $w_{\mathrm{p}}(t)=0.0001 \times[1-\cos (\omega t)]$ (with $f=80 \mathrm{~Hz}$, close to first eigenfrequency) for the uncontrolled Eq. (30) and the controlled configurations Eq. (31)-(33) (relative displacements shown in $(\mathbf{a}, \mathbf{c}$ and $\mathbf{e})$, the bending moment shown in $(\mathbf{b}, \mathbf{d}, \mathbf{f}))$

Figure 3 shows the results if only one of the two terms of the bending moment control solution is considered. The case control-static (magenta and Eq. (32)) is quite interesting from a practical point of view, since no spatial variation of the control voltage is required $V(t)=-4 K_{\mathrm{M}} w_{\mathrm{p}}(t) /\left(e_{31} h b l^{2}\right)$, see Eq. (39). A technological advantageous possibility how to realize other distributions, which depend on the $x$-coordinate, is given in Schoeftner et al. [22]. The results show that only in the lower frequency range, the bending moment and the maximum stress are small: $0.185 \mathrm{Nm}$ is the bending moment and $1.09 \times 10^{7} \mathrm{~N} / \mathrm{m}^{2}$ is the axial stress at the first resonance. This value is half of the tensile stress limit (dash-dot black line). For the second eigenfrequency, the stress even exceeds the stress limit. For the case control-dynamic (grey, Eq. (33)), the results around the first eigenfrequency is similar to the uncontrolled case. Only the second mode of the stress is lowered; nevertheless, the stress value is also higher than the allowable tensile stress. This method is not a suitable alternative for the reduction of the bending moment or of the stress.

\subsection{Transient harmonic response}

Figure 4 shows the transient responses of the beam for $w_{\mathrm{p}}(t)=w_{0}[1-\cos (\omega t)]$. The relative displacement and the bending moments are shown for the uncontrolled and the three controlled configurations, see Eqs. (31)(33). The excitation frequency is $f=80 \mathrm{~Hz}$.

The stationary bending moment is reduced from $0.158 \mathrm{Nm}$ (uncontrolled-blue) to $0.011 \mathrm{Nm}$ (controlledred). It is noted that the suppression of the bending moment is much higher $(-90 \%$, Fig. $4 \mathrm{~b})$ than for the deflection $(-50 \%$, Fig. $4 \mathrm{~b})$, when comparing the relative deflection and the bending moment. This shows that 
a very low bending moment does not necessarily yield very small deflections: the reduction of the deflection and the bending moment are to two different tasks from a control engineering point of view.

Comparing the case control-static with the uncontrolled configuration, we observe also a significant reduction from $0.158 \mathrm{Nm}$ to $0.029 \mathrm{Nm}$, see Fig. $4 \mathrm{~d}$.

As expected from Fig. 3b, the bending moment for the case control-dynamic (Fig. 4b) is hardly influenced as long as $f \ll f^{+} \approx 161 \mathrm{~Hz}$. The stationary amplitude is even slightly increased from $0.158 \mathrm{Nm}$ to $0.175 \mathrm{Nm}$, and a reduction can only be obtained around the second eigenfrequency (cf. Fig. $3 \mathrm{~b}$ and $f \approx 585 \mathrm{~Hz}$ ).

\section{Conclusion}

In the present paper, an open loop control method is presented how to control the bending moment of beam structures by eigencurvature actuation produced by the piezoelectric effect. It is shown that by manipulating the actual bending moment, one can also control the axial stress, which is mainly responsible for the fracture and fatigue of the material, if a certain stress limit is exceeded. After recalling the fundamental equations for piezoelectric beams, a positive definite, time-dependent integral is defined, depending on the error bending moment. The error moment is the difference between the actual moment and the desired moment. By demanding the integral to be zero for all times, conditions for the eigencurvature are found, which also have to satisfy the initial and the boundary conditions. This theory is verified by a simple example, a support-excited beam. The required bending moment is achieved by means of a suitable eigencurvature actuation, which is a function of place and time. Additionally, the maximum axial stress can be significantly decreased in comparison with the uncontrolled support-excited beam.

Acknowledgements Open access funding provided by Johannes Kepler University Linz. J. Schoeftner acknowledges support from the Austrian Science Fund FWF via the Project P 26762-N30.

Open Access This article is distributed under the terms of the Creative Commons Attribution 4.0 International License (http:// creativecommons.org/licenses/by/4.0/), which permits unrestricted use, distribution, and reproduction in any medium, provided you give appropriate credit to the original author(s) and the source, provide a link to the Creative Commons license, and indicate if changes were made.

\section{References}

1. Hetnarski, R.B., Ignaczak, J.: Mathematical Theory of Elasticity. Taylor \& Francis, London (2004)

2. Gurtin, M.E.: The Linear Theory of Elasticity. Encyclopedia of Physics (S.Fluegge, Ed.), VIa/2. Springer, Berlin (1972)

3. Lurie, A.I.: Theory of Elasticity. English translation by A.K. Belyaev. Springer, Berlin (2005)

4. Moheimani, S.O.R., Fleming, A.J.: Piezoelectric Transducers for Vibration Control and Damping. Springer, London (2006)

5. Crawley, E.F.: Intelligent structures for aerospace: a technology overview and assessment. AIAA J. 32, 1689-1699 (1994)

6. Miu, D.K.: Mechatronics: Electromechanics and Contromechanics. Springer, New York (1993)

7. Tzou, H.S.: Multifield transducers, devices, mechatronic systems and structronic systems with smart materials. Shock Vib. Dig. 30, 282-294 (1998)

8. Mura, T.: Micromechanics of Defects in Solids, 2nd edn. Martinus Nijhoff, Dordrecht (1991)

9. Schoeftner, J., Krommer, M.: Single point vibration control for a passive piezoelectric Bernoulli-Euler beam subjected to spatially varying harmonic loads. Acta Mech. 223, 1983-1998 (2012)

10. Krommer, M., Irschik, H.: An electromechanically coupled theory for piezoelastic beams taking into account the charge equation of electrostatics. Acta Mech. 154, 141-158 (2002)

11. Krommer, M., Irschik, H.: A Reissner-Mindlin-type plate theory including the direct piezoelectric and the pyroelectric effect. Acta Mech. 141, 51-69 (2000)

12. Han, L., Wang, X.D., Zuo, M.: The dynamic behavior of a surface-bonded piezoelectric actuator with a bonding layer. Acta Mech. 206, 193-205 (2009)

13. Benjeddou, A., Deü, J.F.: A two-dimensional closed-form solution for the free-vibration analysis of piezoelectric sandwich plates. Int. J. Solids Struct. 39, 1463-1486 (2002)

14. Irschik, H.: A review on static and dynamic shape control of structures by piezoelectric actuation. Eng. Struct. 24, 5-11 (2002)

15. Hubbard, J.E., Burke, S.E.: Distributed transducer design for intelligent structural components. In: Tzou, H.S., Anderson, G.L. (eds.) Intelligent Structural Systems. Kluwer Academic Publishers, Norwell (1992)

16. Hafka, R.T., Adelman, H.M.: An analytical investigation of shape control of large space structures by applied temperatures. AIAA J. 23, 450-457 (1985)

17. Irschik, H., Krommer, M., Belayaev, A.K., Schlacher, K.: Shaping of piezoelectric sensors/actuators for vibrations of slender beams: coupled theory and inappropriate shape functions. J. Intel. Mater. Syst. Struct. 9, 546-554 (1998)

18. Irschik, H., Krommer, M., Pichler, U.: Dynamic shape control of beam-type structures by piezoelectric actuation and sensing. Int. J. App. Electromagn. Mech. 17, 251-258 (2003) 
19. Nader, M.: Compensation of vibrations in smart structures: shape control, experimental realization and feedback control. Doctoral thesis Johannes Kepler University Linz. Trauner, Linz, Austria (2007)

20. Irschik, H., Pichler, U.: An extension of Neumann's method for shape control of force-induced elastic vibrations by eigenstrains. Int. J. Solids Struct. 41, 871-884 (2004)

21. Schoeftner, J., Buchberger, B., Brandl, A., Irschik, H.: Theoretical prediction and experimental verification of shape control of beams with piezoelectric patches and resistive circuits. Compos. Struc. 133, 746-755 (2015)

22. Schoeftner, J., Buchberger, B., Irschik, H.: Static and dynamic shape control of slender beams by piezoelectric actuation and resistive electrodes. Compos. Struct. 111, 66-74 (2014)

23. Schoeftner, J., Buchberger, B., Benjeddou, A.: Slender piezoelectric beams with resistive-inductive electrodes modeling and axial wave propagation. Smart Struct. Syst. 18(2), 335-354 (2016)

24. Austin, F., Rossi, M.J., Van Nostrand, W., Knowles, G.: Static shape control of adaptive wings. AIAA J. 32, 1895-1901 (1994)

25. Agrawal, B.N., Treanor, K.E.: Shape control of a beam using piezoelectric actuators. Smart Mater. Struct. 8, 729-740 (1999)

26. Irschik, H., Krommer, M., Zehetner, C.: Displacement tracking of pre-deformed smart structures. Smart Struct. Syst. 18, $139-154$ (2016)

27. Irschik, H.: Generation of transient desired displacement or stress fields in force loaded solids and structures by smart actuation. In: Book of Abstracts of XXXV Summer School Advanced Problems in Mechanics (APM 2007), June 20-28th, Repino, Saint-Petersburg, Russia (2007)

28. Irschik, H., Gusenbauer, M., Pichler, U.: Dynamic stress compensation by smart actuation. In: Proceedings of SPIE on Smart Structures and Materials 2004: Modeling, Signal Processing, and Control (R. C. Smith, Ed.), 5383, paper no. 386, March 20-28th, San Diego, CA, 2004, March 15-18 (2004)

29. Ziegler, F.: Mechanics of Solids and Fluids, 2nd edn. Springer, New York (1998)

30. Schoeftner, J., Buchberger, B., Benjeddou, A.: Transverse dynamics of slender piezoelectric bimorphs with resistive-inductive electrodes. Smart Struct. Syst. 18(2), 355-374 (2016)

31. Schoeftner, J., Buchberger, G.: A contribution on the optimal design of a vibrating cantilever in a power harvesting application-optimization of piezoelectric layer distributions in combination with advanced harvesting circuits. Eng. Struct. 53, 92-101 (2013)

32. Website of a manufacturer for piezocreamic patches. http://www.piezo.ws/piezoelectric_actuator_tutorial/Piezo_Design_ part3.php (June 6th, 2016) 